\title{
Innovative patterning method for modifying few-layer MoS2 device
}

geometries

Fernando Jiménez Urbanos ${ }^{1 \mathrm{a}}$, Andrés Black ${ }^{\mathrm{a}, \mathrm{b}}$, Ramón Bernardo-Gavito ${ }^{\mathrm{c}}$, Manuel R. Osorio ${ }^{\mathrm{a}}$, Santiago Casado ${ }^{\mathrm{a}}$, Daniel Granados ${ }^{\mathrm{a}}$

a. Fundación IMDEA Nanociencia, C/Faraday, 9, 28049, Madrid, Spain

b. Departamento de física de la materia condensada, Universidad Autónoma de Madrid, Cantoblanco, 28049, Madrid, Spain

c. Physics Department, Lancaster University, LA1 4YB, Lancaster, United Kingdom

\section{ABSTRACT:}

When mechanically exfoliated two-dimensional (2D) materials are used for device applications, their properties strongly depend on the geometry and number of layers present in the flake. In general, these properties cannot be modified once a device has been fabricated out of an exfoliated flake. In this work we present a novel nano-patterning method for 2D material based devices, Pulsed eBeam Gas Assisted Patterning (PEBGAP), that allows us to fine tune their geometry once the device fabrication steps have been completed.

Keywords: 2D materials, PEBGAP, $\mu$ Raman, sulfur vacancies

\section{INTRODUCTION:}

Transition metal dichalcogenides (TMDCs) such as $\mathrm{MoS}_{2}$, which are considered candidates for next generation optoelectronic technology, consist of discrete two-dimensional (2D) layers bound together by weak van der Waals forces. For that reason, flakes can be exfoliated easily by the Scotch-tape method [1]. These flakes exhibit distinctive thickness dependent variations in their physical properties [2-5].

The band structure of $\mathrm{MoS}_{2}$ varies with the multilayer thickness, going from a direct bandgap in single layer (SL) with a value of $1.8 \mathrm{eV}$ to an indirect bandgap of $1.2 \mathrm{eV}$ in bulk [6,7]. The SL semiconductor device exhibits unique optical properties, including strong photoluminescence (PL) [8], valley polarization $[9,10]$ and strongly charged excitons [11].

It is important to note that fabricating $\mathrm{MoS}_{2}$ electronic devices from mechanically exfoliated flakes is a complex process. In all cases, device size is limited by the geometry of the initial $2 \mathrm{D}$ flake, even when a deterministic stamping method is employed [12]. For this reason, tailoring the device after lithographical processing is of interest, regardless of its original geometry. In the past, interacting electron and ion beams have been used with etching gases to pattern different substrates. Reactions of $\mathrm{Si}, \mathrm{SiO}_{2}$ and $\mathrm{Si}_{3} \mathrm{~N}_{4}$ substrates with $\mathrm{XeF}_{2}, \mathrm{~F}_{2}$ and $\mathrm{Cl}_{2}$ gases were experimentally studied [13]. A similar approach was used with $\mathrm{MoS}_{2}$ placing it in a $\mathrm{XeF}_{2}$ atmosphere at high pressure [15]. This process, however, etched the entire exposed surface of the flake, without in situ control of flake geometry. Laser thinning of $\mathrm{MoS}_{2}$ has been studied [14]. In this case, a control on the flake geometry was reached with size limitation.

In this work our goal is to produce nanostructures in situ,without the need for further lithographical processing. With that in mind, instead of having a two-step post fabrication process consisting of lithography and subsequent etching, (1) the Pulsed Electron-Beam Gas Assisted Patterning (PEBGAP) method combines these two steps into one.This novel nanopatterning method, which uses a carrier gas and an electron beam to laterally etch the channel of an already fabricated $\mathrm{MoS}_{2}$ device into specific geometries. The effect of this fabrication method on the electronic properties of the device will be studied.

1 fernando.jimenez@imdea.org; http://nanoscience.imdea.org/ 


\section{EXPERIMENTAL:}

$\mathrm{MoS}_{2}$ flakes were exfoliated onto a degenerately $\mathrm{n}$-doped silicon wafer capped with a $285 \mathrm{~nm} \mathrm{SiO} 2$ layer. Patterning of electrical contacts is done using laser beam optical lithography, followed by thermal evaporation of chromium $(10 \mathrm{~nm})$ and gold $(70 \mathrm{~nm})$ resulting in a back-gated field effect transistor geometry. After the contact definition and the liftoff, an

annealing is then performed in an argon/hydrogen atmosphere at $300^{\circ} \mathrm{C}$ to remove resist residues and decrease contact resistance. Electrical transport measurements were carried out in ambient using a probe station and a Keithley 4200 SCS parameter analyzer. Scanning Raman mapping measurements were also carried out in ambient, using a 40x magnification objective, and exciting with a $488 \mathrm{~nm}$ Ar laser.

Following the initial characterization of the devices, PEBGAP nano-patterning is used to alter device geometries. This method consists on the combination of a pulsed electron beam and a micro gas injection system. First, the sample is introduced into the Ultra High Resolution Scanning Electron Microscope. Figure 1 shows a schematic view of the process. As shown in Figure 1a, the gas enters the chamber through a small nozzle and adsorbs on the device surface. The eBeam is then pulsed and scanned over the device surface as shown in Figure 1b. The electrons dissociate the gas, causing the fluorine atoms to etch the desired zones of the device, as shown in Figure 1c.

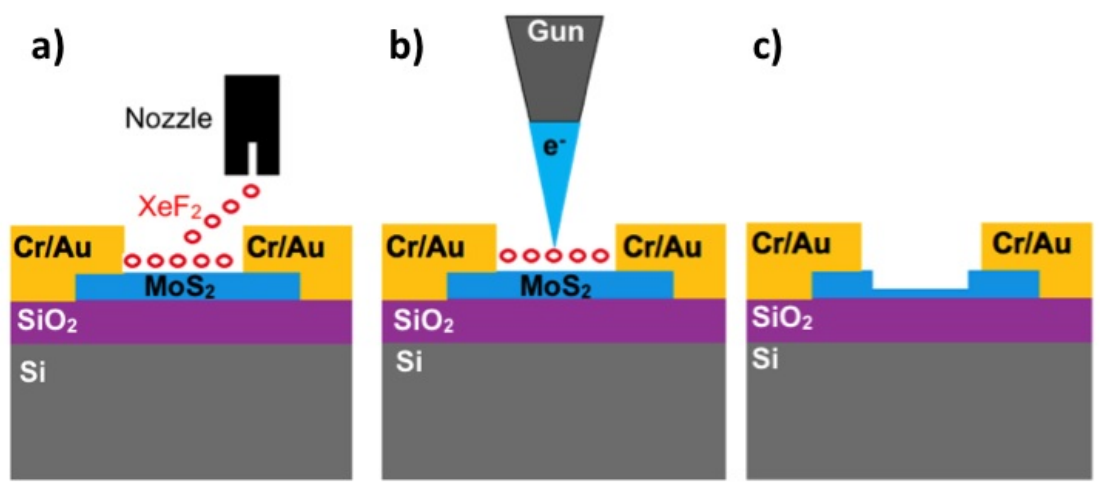

Figure 1. a) Schematic of the gas entering the chamber through the nozzle and adsorbing on the device surface. b) Schematic of the focused eBeam scanning the sample. c) Final etched device.

After PEBGAP nano-patterning, the devices were characterized again by electrical, AFM and $\mu$ Raman means. Following this characterization, the devices were annealed at $300{ }^{\circ} \mathrm{C}$ during $2 \mathrm{~h}$ and finally characterized.

\section{RESULTS:}

Figure 2 shows a $\mathrm{MoS}_{2}$ device before and after PEBGAP. Figure 2.a) shows a device width of $2.5 \mu \mathrm{m}$. After PEBGAP, a funnel geometry of approximately $250 \mathrm{~nm}$ has been created as shown in Figure $2 \mathrm{~b}$. A light shadow in the image of Figure $2 b$, surrounding the funnel device, corresponds to the regions of the original device that were etched. The discoloration is due to a slight etching of the underlying $\mathrm{SiO}_{2}$ substrate, as will be confirmed later in AFM measurements.
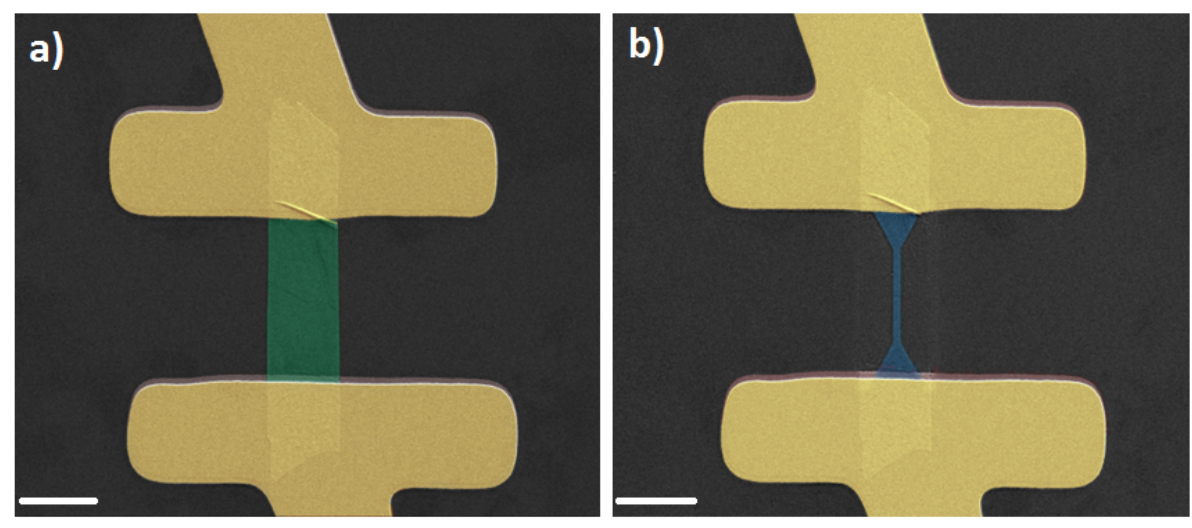
Figure 3a shows an IV curve at 0 back gate voltage before and after PEBGAP. The device was initially $2.5 \mu \mathrm{m}$ wide and was narrowed to $250 \mathrm{~nm}$. The green IV curve shown in figure 3.a) corresponds to the "intrinsic" device, where a quasiohmic contact can be observed. In addition, the blue IV curve in Figure 3a, which corresponds to the device after PEBGAP and annealing, shows a diode-like curve. Apart from the change in curve shape, it also is important to mention that there is a large decrease in the current after PEBGAP. The green curve of Figure $3 \mathrm{a}$ is in the $\mu \mathrm{A}$ range, while blue curve is in the nA range. The decrease in channel width after PEBGAP is partially responsible, and should reduce the current to approximately $10 \%$ of its initial value.

In addition, doping changes may be playing a role. The transistor curve shown in figure 3.b) reveals that the OFF and ON states of the device before and after PEBGAP are not in the same place. Thus, when measuring at 0 gate voltage, the intrisic device is in the ON state while the PEBGAP device is in the OFF state with values of the current in the nA. A threshold gate voltage shift from $-65 \mathrm{~V}$ for the intrinsic device to $55 \mathrm{~V}$ for the PEBGAP device indicates a p-type doping.
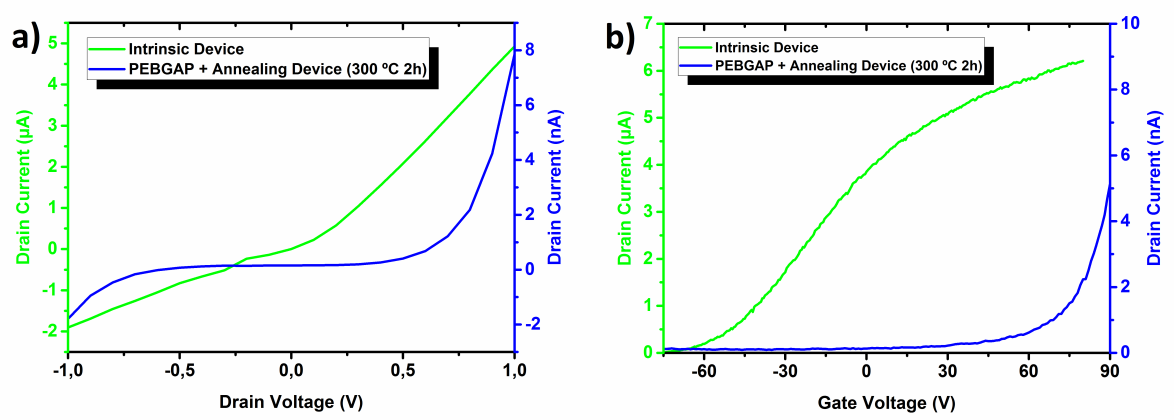

Figure 3. a) IV curve of the intrinsic device (green) and the patterned device (blue). The intrinsic device shows a quasi-ohmic contact while the PEBGAP device shows a diode-type curve. Current scales are different due to two phenomena (narrowing of the

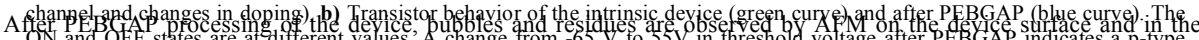

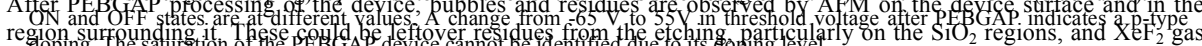
bubbles trapped within the $\mathrm{MoS}_{2}$ device. By annealing the device, a much cleaner surface is obtained, as shown in Figure $4 \mathrm{~b}$. In addition, a change in device height from 10 to $6 \mathrm{~nm}$ is measured before and after annealing. This change in 
the device height could indicate the disappearance of trapped $\mathrm{XeF}_{2}$ gas within the $\mathrm{MoS}_{2}$ layers of the device, and/or the elimination of etching residues between the device and the substrate.. Interaction between the XeF2 gas and the $\mathrm{MoS}_{2}$ during PEBGAP, or more likely with the trapped gas bubbles during high temperature annealing, could be generating sulfur vacancies within the $\mathrm{MoS}_{2}$ device channel [REFERENCES HERE?]. Sulfur vacancies are known to have a pdoping effect [OR REFERENCES HERE], as confirmed by experiments using phosphorous plasma immersion ion implantation to create them within a $\mathrm{MoS}_{2}$ device [20]. This would explain the p-doping observed in the electrical measurements in Figure 3b. Figure 4 also reveals a slight etching of the $\mathrm{SiO}_{2}$ substrate in the regions where the PEBGAP was carried out, as was previously observed in the SEM images of Figure 2.
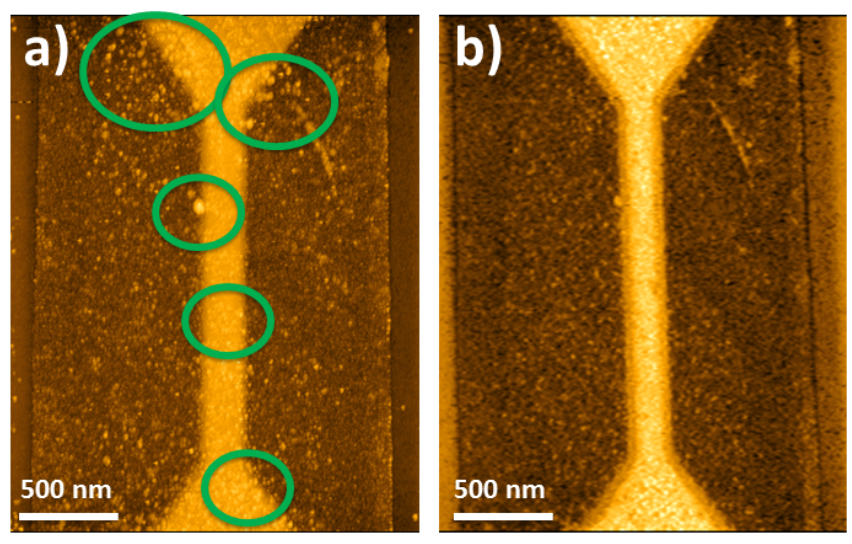

Figure 4. a) AFM image of the processed device before annealing. Green circles round some observed gas bubbles. b) AFM image of the processed device after annealing. A decrease in height profile is observed going from $10 \mathrm{~nm}$ height before annealing to $6 \mathrm{~nm}$ height after annealing.

$\mu$ Raman characterization of devices before and after PEBGAP give information about structural and chemical composition changes. An intensity mapping of the $\mathrm{E}_{2 \mathrm{~g}}^{1} \mathrm{MoS}_{2}$ characteristic peak before and after PEBGAP is shown in Figure 5a. The peak's presence after PEBGAP indicates that the post-processing method does not alter the main chemical composition of the $\mathrm{MoS}_{2}$ device. The $\mathrm{A}_{1 \mathrm{~g}}$ peak shows the same behavior as the $\mathrm{E}_{2 \mathrm{~g}}$ peak, and for that reason only one peak is shown in Figure 5a. Figure 5b shows a full width at half maximum (FWHM) $\mu$ Raman mapping of the $E_{2 \mathrm{~g}}^{1}$ peak before and after PEBGAP, revealing a change of the FWHM from $3.5 \mathrm{~cm}^{-1}$ before PEBGAP to $5.5-6 \mathrm{~cm}^{-1}$ after PEBGAP in the entire flake. The same features are observed for the $A_{1 \mathrm{~g}}$ peak.

Figure 6 represent a $\mu$ Raman mapping corresponding to the difference in position between the two $\mathrm{MoS}_{2}$ characteristic peaks (Peak Position Difference, PPD) measured in $\mathrm{cm}^{-1}$ before and after PEBGAP, showing a change in the PPD from 23-24 $\mathrm{cm}^{-1}$ before PEBGAP to $21-22 \mathrm{~cm}^{-1}$ after PEBGAP.

These changes after PEBGAP in FWHM and PPD shown in Figure 5b and Figure 6 respectively could indicate a change in the doping as observed in [24,25], where they attribute changes in $\mu$ Raman parameters to changes in the doping. Specifically, it was observed an increase in the FWHM and a reduction in the PPD as shown in our results.

\section{a) BEFORE PEBGAP}

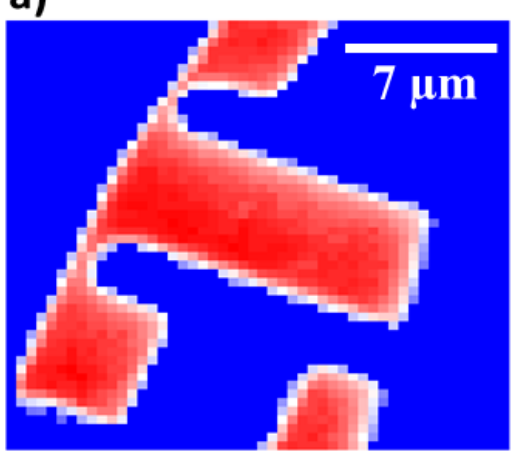

Raman Intensity (a.u.)

b)

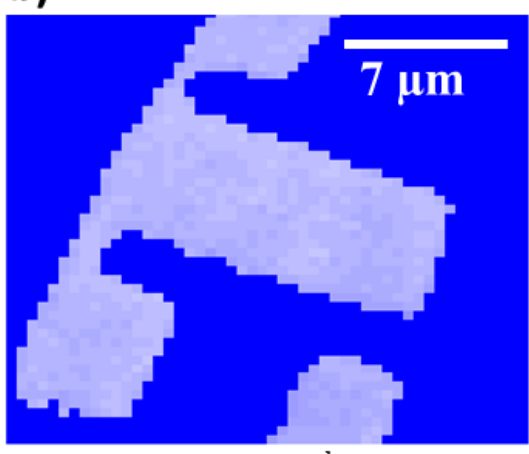

$\operatorname{PPD}\left(\mathrm{cm}^{-1}\right)$

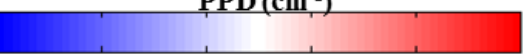

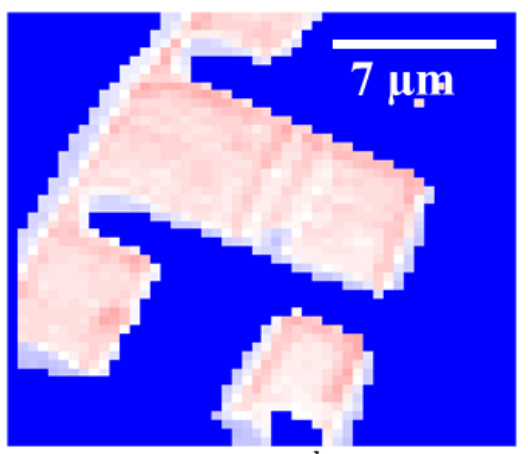

$\operatorname{PPD}\left(\mathrm{cm}^{-1}\right)$
AFTER PEBGAP
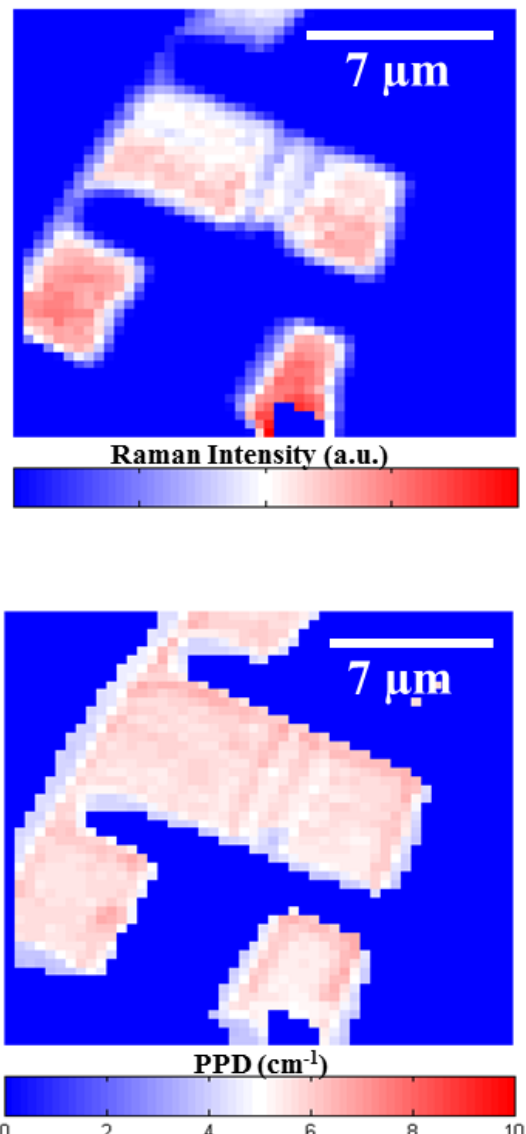

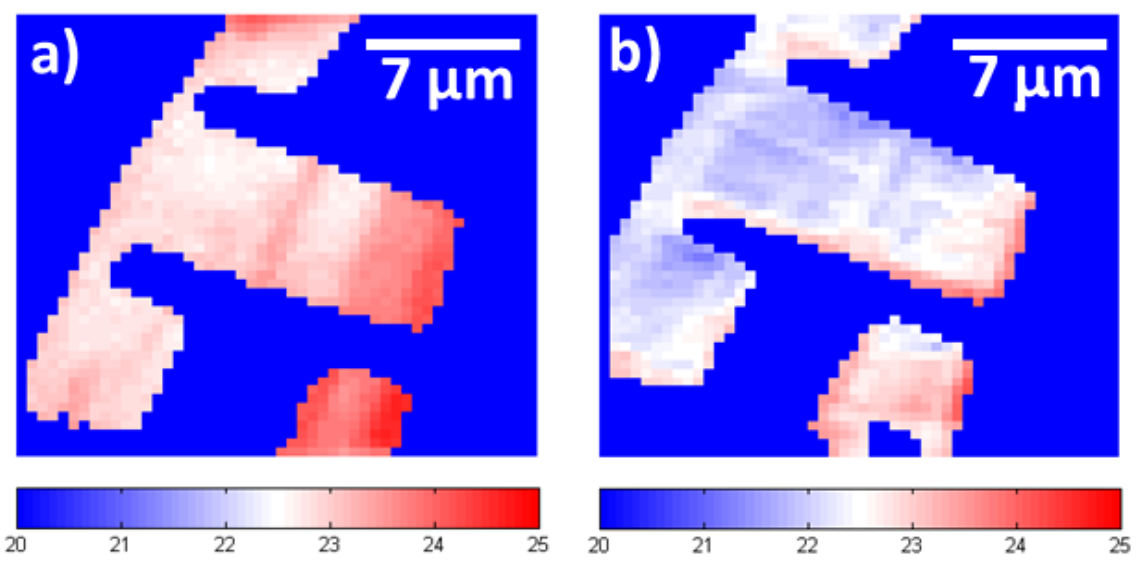
Figure 6. a) $\mu$ Raman PPD mapping before PEBGAP. PPD value is around $23-24 \mathrm{~cm}^{-1}$. b) $\mu$ Raman PPD mapping after PEBGAP. PPD value is around $21-22 \mathrm{~cm}^{-1}$. A shift to lower values between $2-3 \mathrm{~cm}^{-1}$ is observed after PEBGAP indicating, probably, a change in doping.

\section{CONCLUSIONS}

The experiments here reported have shown that PEBGAP is a tool that allows tailoring device geometries after fabrication, combining lithographic patterning and subsequent etching into a single step.

After PEBGAP the, device's electrical characteristics are significantly changed,showing a transition from n-type to $\mathrm{p}$ type doping. After PEBGAP, AFM characterization revealed the presence of gas bubbles and etching residues on the device surface and possibly between the layers of the $\mathrm{MoS}_{2}$. These were eliminated by a subsequent high temperature annealing, cleaning the device and the area surrounding it, as well as reducing the height of the device itself. We speculate that the high temperature annealing may cause the $\mathrm{MoS}_{2}$ to interact with the trapped gas and residues, creating sulfur vacancies in the device channel which may be responsible for the observed p-doping, as reported by other authors.

Doping is also revealed by $\mu$ Raman spectroscopy, which shows a change in the FWHM and PPD values after PEBGAP.

\section{REFERENCES:}

[1] Novoselov, K.S. et al. PNAS USA 2005, 102, 10451- 10453

[2] Sina Najmaei et al. ACS Nano 2014, 8, 7930-7937

[3] Xiao Huang et al. Chem. Soc. Rev. 2013, 42, 1934

[4] D.Kufer and G.Konstantanos. Nano Lett. 2015, 15, 7307-7313 
[5] Jungwook Choi et al. ACS Nano 2016, 10, 1671-1680

[6] Mak, K.F et al. Phys. Rev. Lett. 2010, 105, 136805

[7] Kam, K.K. et al. J. Phys. Chem. 1982, 86, 463-467

[8] Splendiani, A. et al. Nano Lett. 2010, 10, 1271-1275

[9] Mak, K.F. et al. Nat. Nanotechnol. 2012, 7, 494-498

[10] Zeng, H. et al. Nat. Nanotechnol. 2012, 7, 490-493

[11] Ross, J.S. et al. Nat. Commun. 2013, 4, 1474

[12] Castellanos-Gomez, A. et al. 2D Materials 2014, 1, 025001

[13] Coburn, J. et al. Journal of App. Phys. 1979, 50, 3189- 3196

[14] Castellanos-Gomez, A. Nano Lett. 2012, 12, 3187-3192

[15] Huang, Y. et al. Nano Research 2013, 6, 200-207

[16] Hong Li et al. Adv. Funct. Mater. 2012, 22, 1385-1390

[17] Ramon paper PEBGAP

[19] Carvalho, A. et al. Phys. Rev. B: Condens. Matter Mater. Phys. 2014, 89, 081406-081410.

[20] Nipane, A. et al. ACS Nano, 2016, 10, 2128-2137

[21] Dong Min Sim et al. ACS Nano, 2015, 9, 12115-12123

[22] Brahim Akdim et al. Nanotechnology, 2016, 27, 185701

[23] Jinhua Hong et al. Nature Commun. 2015, 520, 656-660

[24] Chakraborty, B. et al. Phys. Rev. B, 2012, 85, 161403

[25] William M. Parkin et al. ACS Nano, 2016, 10, 4134-4142 